\title{
Rescuing protein homeostasis
}

The maintenance of protein homeostasis (proteostasis) is vital for cell function and involves a tightly regulated network of pathways controlling the synthesis, folding, transport and degradation of proteins. Loss of proteostatic control can lead to the accumulation and aggregation of misfolded proteins - features that are associated with numerous conditions including metabolic diseases, cancer and neurodegenerative disorders. Now, Calamini and colleagues describe a series of novel small-molecule proteostasis regulators that activate the heat shock response (HSR) and restore protein homeostasis in various models of protein conformational diseases.

Crucial to proteostasis are the cytosolic HSR and the unfolded protein response (UPR), which are induced under conditions of cellular

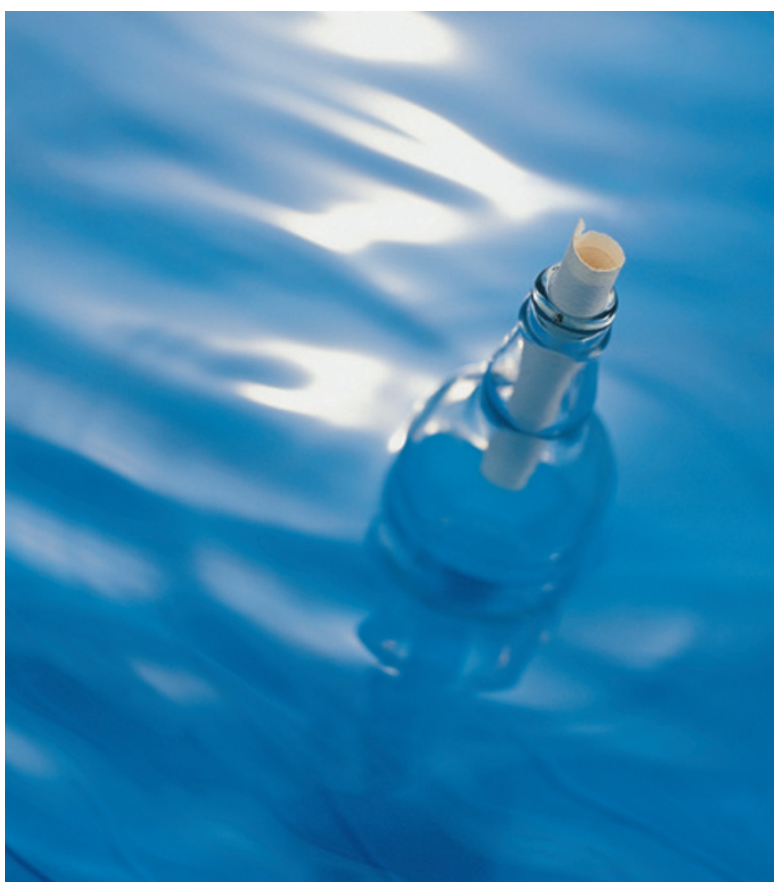

stress and act to restore protein balance in the cytoplasm and endoplasmic reticulum, respectively. The HSR is governed by a family of heat shock factors (HSFs) - primarily HSF1, which promotes the expression of genes encoding molecular chaperones, such as the heat shock proteins (HSPs), that guide the conformation of proteins during biogenesis and prevent their misfolding and aggregation. Enhancing the activity of HSF1 and the concentrations of molecular chaperones has previously been shown to restore proteostasis in several disease models. With this in mind, Calamini and colleagues set out to identify novel small-molecule proteostasis regulators.

To do this, the authors developed a human cell-based high-throughput screen to identify activators of the HSR. Using two independent primary screens encompassing a total of 900,000 compounds, they obtained and confirmed 263 positive hits. Clustering active compounds by structure resulted in 233 hits grouped into seven clusters, and identified three common scaffolds across the two primary screens. Fourteen compounds were selected as representative small-molecule 'proteostasis regulators' for subsequent studies.

Further in vitro experiments confirmed that the newly identified molecules activated the HSR; they induced mRNA and protein expression of several HSPs in an HSF1-dependent manner. Analysis of the gene signatures of the proteostasis regulators revealed that some molecules activated additional stressresponsive proteostasis network pathways, including the UPR and the antioxidant stress response. Of the five compounds that were shown to most strongly activate HSF1, three significantly protected cells from death induced by severe heat shock and from apoptosis induced by oxidative stress.

Next, the authors assessed whether the proteostasis regulators could restore proteostasis in two cellular models of conformational diseases - Huntington's disease and cystic fibrosis. Indeed, the compounds reduced aggregate formation of a mutant huntingtin protein containing a polyglutamine repeat, and rescued trafficking and cell surface expression of a mutant cystic fibrosis transmembrane conductance regulator in the respective disease models. Furthermore, in a Caenorhabditis elegans model of polyglutamine diseases, the proteostasis regulators reduced the aggregation of expanded polyglutamines and ameliorated aggregation-associated toxicity, restoring motility to close to that of control animals.

In contrast to current known small-molecule activators of the HSR, these compounds did not act by causing protein misfolding, proteasome inhibition or HSP90 inhibition.

These findings support further investigation of small-molecule proteostasis regulators for the potential treatment of multiple proteopathies, and the novel molecules identified in this study represent promising first-generation tool compounds.

Sarah Crunkhorn

ORIGINAL RESEARCH PAPERS Calamini, B. et al. Small-molecule proteostasis regulators fo protein conformational diseases. Nature Chem. Biol. 25 Dec 2011 (doi:10.1038/nchembio.763) FURTHER READING Neef, D. et al. Heat shock transcription factor 1 as a therapeutic target in neurodegenerative diseases. Nature Rev. Drug Discov. 10, 930-944 (2011) 